\title{
Attentionally splitting the mass distribution of hand-held rods
}

\author{
GREGORY BURTON and M. T. TURVEY \\ Center for the Ecological Study of Perception and Action, \\ University of Connecticut, Storrs, Connecticut
}

\begin{abstract}
Two experiments on the length-perception capabilities of effortful or dynamic touch differed only in terms of what the subject intended to perceive, while experimental conditions and apparatus were held constant. In each trial, a visually occluded rod was held as still as possible by the subject at an intermediate position. For two thirds of the trials, a weight was attached to the rod above or below the hand. In Experiment 1, in which the subject's task was to perceive the distance reachable with the portion of the rod forward of the hand, perceived extent was a function of the first moment of the mass distribution associated with the forward portion of the rod, and indifferent to the first moment of the entire rod. In Experiment 2, in which the task was to perceive the distance reachable with the entire rod if it was held at an end, the pattern of results was reversed. These results indicate the capability of selective sensitivity to different aspects of a hand-held object's mass distribution, without the possibility of differential exploration specific to these two tasks. Results are discussed in relation to possible roles of differential information, intention, and self-organization in the explanations of selective perceptual abilities.
\end{abstract}

A number of recent experiments have been directed at the ability to perceive the extents of hand-held rods through effortful or dynamic touching (Gibson, 1966)-the kind of touching that involves muscular exertion in conjunction with stimulation of the skin and joints, as in the wielding of implements and tools, and the hefting and throwing of objects. The major finding of these experiments-in which rods were wielded with manipulations of rod length, hand position, mass distribution, and frequency of wielding-is that variation in perceived extent is constrained by variation in moment of inertia. The more inertia a rod has for rotation, the greater its perceived extent (Solomon \& Turvey, 1988; Solomon, Turvey, \& Burton, 1989a, 1989b). It is important, however, that although moment of inertia fully constrains the perception of extent, moment of inertia is not the property perceived (just as it is often argued that humans perceive depth through retinal disparity but do not perceive retinal disparity). A person is aware of object length or reachable distance, and when the hand-held object has a uniform density, perceived extents can match actual extents. For example, in Experiment 2 of Solomon and Turvey (1988), actual rod lengths were $30.5,45.7,61.0,76.2$, and $91.4 \mathrm{~cm}$, and perceived rod lengths were, respectively, $31.9,46.4$, $65.2,80.9$, and $92.4 \mathrm{~cm}$.

This research was supported by NSF Grant BNS-8720144. The authors would also like to acknowledge the contribution of discussions with Robert Shaw, Tin-Cheung Chan, and H. Yosef Solomon, as well as the helpful comments of Myron Braunstein and three anonymous reviewers on an earlier version of the manuscript. M. T. Turvey is also at Haskins Laboratories. Correspondence should be addressed to Gregory Burton, Department of Psychology, McQuaid Hall, Seton Hall University, South Orange, NJ 07079.
A significant variation of this result in effortful or dynamic touching is that, for a given position of the hand on the rod, and with the rod grasped tightly so that the rod does not move relative to the hand, a person can perceive selectively either the whole rod extent or the partial rod extent forward of the hand. Perception of the whole extent varies with the total moment of inertia of the rod relative to the position of grasp; perception of the partial extent varies with the moment of inertia of the rod segment forward of the position of grasp (Solomon \& Turvey, 1988; Solomon et al, 1989b). The only difference between these partial and whole rod conditions is what the subject intends ("that I perceive the extent of rod forward of my hand" "vs. "that I perceive the extent of the whole rod") and, thereby, what the subject focuses attention upon ("the segment of rod forward of the hand" vs. "the whole rod"). Because perception of extent in the partial condition is related to the rotational inertia of the partial rod and indifferent to the rotational inertia of the whole rod, the phenomenon expresses an intentional/ attentional splitting of the rod's mass distribution. The phenomenon is all the more remarkable, given that, even when perceiving partial extent by wielding, the deformation of the tissues (skin, muscles, tendons, ligaments) of the hand and arm is induced by the rigid body motion of the whole (and not the partial) rod in conjunction with muscular forces. The patterning of deformation peculiar to motions of the partial rod extent is embedded in the patterning of deformation due to motions of the whole rod.

The splitting of the mass distribution by attention in effortful or dynamic touching shares similarities with several phenomena in vision, phenomena found in situations that require the observer to attend selectively to one of two 
displays occupying the same spatial region. This selective looking paradigm was first introduced by Neisser and Becklen (1975), whose subjects encountered no difficulty in watching one of two videotaped events (a ball game and a hand game) superimposed spatially at the same optical distance. (In other paradigms in visual attention, such as that of Driver and Bayliss [1989], there are intimations of constraints on the dimensions along which displays can be selectively separable.) Investigations by Tsal (1984) have shown that when the wings-in and wings-out versions of the Müller-Lyer figures are superimposed, the horizontal line is perceived as less extensive when the inside pointing wings are attended to than it is when the outside pointing wings are attended to. Similarly, if the superimposed Müller-Lyer figures are distinguished by color, then the perceived extent of the horizontal line depends on which of the two colored versions (wings in or wings out) receives attention (Goryo, Robinson, \& Wilson, 1984). The classic ambiguous figures of the bird/plane and duck/ rabbit provide a further illustration of the selective looking phenomenon. Which of the two depictions is seen (e.g., duck or rabbit) depends on how attention is distributed over the ambiguous figure, a fact that is demonstrated by the more rapid detection of targets in the region of the figure critical to the current perception relative to when they appear in the region critical to the noncurrent, alternative perception (Tsal \& Kolbert, 1985). Of particular relevance to the attentional partitioning of the mass distribution in effortful or dynamic touching is the demonstration by Nicoletti and Umilta (1989) that directing visual attention to a particular spatial location of a display results in a left-right division of the display about the focus of attention, with significant behavioral consequences. Specifically, stimulus-response compatibilities can be defined and observed in terms of the attentioninduced partitioning; right-side responses to targets to the right of the attentive focus and left-side responses to targets to the left of the attentive focus are faster than rightside responses to left-side targets and left-side responses to right-side targets.

With respect to perception of the lengths of occluded hand-held rods, it has been shown that this facility extends to the limiting case of effortful or dynamic touching, when free variation in wielding is eliminated by the requirement of holding the rod still. This fact was demonstrated originally by Hoisington (1920) in Titchener's laboratory at Cornell, and it has been elaborated by Burton and Turvey (1990). These investigators found that the perceived lengths of wooden rods, held at one end but neither seen nor swung, were very close to their actual lengths. For rod lengths of $46,61,76,91,107$, and $121 \mathrm{~cm}$, the average perceived lengths were, respectively, $41,58,77,91,109$, and $111 \mathrm{~cm}$. Manipulation of the number and positions of masses attached to the rods indicated that, under conditions of minimal effortful touching, variations in perceived extent were constrained primarily by variations in the static moment of the mass distribution, namely, $M R$ where $M$ is rod mass and $R$ is the position vector of the center of rod mass. The moment of inertia proved contributory, but to a lesser degree. If a rod could be held absolutely motionless, then resistance to rotational acceleration would be undefined and its contribution to perceived extent would be nil. In practice, the situation of holding without wielding is a condition of minimized wielding; small, but not always negligible, motions are possible. It is nonetheless clear that the significant independent variable for perceiving without free wielding is not the same as that for perceiving with free wielding. As noted above, in free wielding the relevant independent variable is the moment of inertia; and direct comparisons of the holding and free wielding conditions yield opposite patterns of dependency on the static moment and moment of inertia (Burton \& Turvey, 1990).

The static moment and the moment of inertia are, respectively, the first and second moments of the mass distribution of an object. If $m_{i}$ is the mass of particle $i$ and $r_{i}$ is its position vector relative to an arbitrary pivot point or axis, then $m_{i} r_{i}$ is the particle's static moment and $m_{i} r_{i}^{2}$ is the particle's inertia to rotation about that point. The static moment and the moment of inertia of the whole object about the pivot point are given by $\int m_{i} r_{i}$ and $\int m_{i} r_{i}^{2}$, respectively. For an object not to turn about a pivot point due to the twisting force $\boldsymbol{M g}$ acting at $\boldsymbol{R}$ (where $g$ is the constant acceleration due to gravity), a torque $N$ equal and opposite must be provided at the pivot point, hence

$$
N=M R \times g .
$$

In comparison, in order to rotate an object about a pivot point, a torque $N$ must be provided such that

$$
N=I d \omega / d t,
$$

where $I$ is the inertia tensor and $\omega$ is the angular velocity vector.

It seems, therefore, that in the holding and the wielding of a rod, tissues of the body are deformed by torques that are proportional to invariant characteristics of the rod (the moments of the mass distribution) relative to the pivot point. That rod extents can be perceived reliably under the two conditions, and with comparable degrees of accuracy, may be taken to mean that they share a common informational basis despite the distinction between them in the relevant mechanical variable. That is to say, in both cases, perceived extent is potentially specific to information of one and the same kind. Following Gibson (1979), information is said to be contained in structured arrays of energy distributions; such a structured array consists of different magnitudes of a given quantity (e.g, light intensity, rotational inertia) in different directions. For haptic perception, it has been hypothesized that information is contained in structured arrays of tissue strains (or strain rates); such an array comprises different magnitudes of strain (or strain rates) in different directions (Turvey, Solomon, \& Burton, 1989). According to this hypothesis, the structuring of strains by the torques of holding and wielding contains an invariant patterning specific to rod extent. Stated differently, the hypothesis is that the first 
and second moment invariants of the mass distribution causally entail the same invariant of tissue deformation.

Therefore, awareness of rod extent is constrained in similar ways under conditions of holding (minimal effortful touching) and under conditions of wielding. As noted above, a particularly important result concerning wielding is that perceiving can be selective; for a given handheld rod, either the whole extent of the rod or the extent of the part in front of the hand can be reported. Presumably, selective effortful touching rests on (1) a differentiation within the deformation pattern-the structured array of strains-of deformation qualities specific to the rotational inertias of the whole and parts, and (2) a facility to attend to the deformation quality specific to the whole or a part (Solomon et al., 1989b). Because the wielding condition permits the observer to vary perceptual activity - he or she can wield in many varied configurations at many varied rates-it is plausible to assume that one manner of wielding reveals the information that specifies whole extent and another manner reveals the information that specifies a partial extent. Given this assumption, the relevant information and the attentive process are realized and defined, respectively, by a particular style of exploratory behavior. If this is so, we should not expect to find a comparable ability of selectively perceiving whole and partial extents under conditions of minimal effortful touching, when a rod is merely held and there is no opportunity to bring about marked variations in the torque patterns. For a comparable capability of attentionally splitting the mass distribution to be evident in minimal effortful touching, it would have to be the case that the static torque patterns the tissue deformation simultaneously in ways specific to the whole and partial extents, and that haptic attention to these different kinds of information could be achieved covertly (i.e., without external variations in exploration, such as eye movements; see Gibson, 1966, p. 51), as well as overtly. Similarly, some kinds of selective looking documented above (e.g., Nicoletti \& Umiltà, 1989) have been achieved covertly; the "spotlight phenomenon" in vision (see review by Johnston \& Dark, 1986) is a canonical example of covert attention.

In two experiments, we investigated the ability to partition attentionally the mass distribution of a rod under the conditions of holding without wielding. These experiments paralleled those of Solomon et al. (1989b), for the case of free wielding. We manipulated the places along the rods at which they were held and the positions along the rods of an additional, attached mass. For a rod of a given length, these manipulations introduced variations in the magnitude of the static moment $M R$. If there is a basis in the induced tissue deformation for distinguishing whole and partial extents, and if this informational distinction can be attended to covertly, we should see perception of whole extent determined by the static moment of the whole rod about the given pivot point, and perception of the partial extent determined by the static moment of the part of the rod forward of the hand. That is to say, we should see an attentional splitting of the first moment of the mass distribution. Importantly, the success of attentional partitioning was measurable in these experiments. It was given, for example, by the relative proportions of variance of perceived partial extent accounted for by partial static moment and total static moment. If the material distribution of the hand-held object anterior to the grasp position can be separated off perfectly from that posterior to the grasp position, the variance accounted for by the full-length static moment should be zero.

\section{METHOD}

\section{Subjects}

Eight students at the University of Connecticut participated in Experiment 1 in partial fulfillment of a course requirement. Seven of the subjects were females; every subject was right-handed. In Experiment 2, 6 people from the same population served as subjects; all were right-handed females.

\section{Materials}

Nine wooden rods (radius $.64 \mathrm{~cm})$, three rod lengths $(.610, .762$, and $.914 \mathrm{~m})$ and two hand positions $(H=1 / 2 \mathrm{~L}$ and $H=3 / 4 \mathrm{~L}$, where $L$ is rod length) were used in the two experiments. These hand positions meant that either a half or a quarter of a given rod's length was forward of the wrist. The density of each rod was approximately $630 \mathrm{~kg} / \mathrm{m}^{3}$; the actual density was slightly different for each rod, and the exact density was used for calculations of mechanical variables. To one rod of each length was attached a weight of approximately $.06 \mathrm{~kg}$ (again, the masses of the weights differed slightly, and the exact figures were employed in calculations) at a distance of $1 / 8 \mathrm{~L}$ from the proximal end of the rod. To one rod of each length was attached a weight of the same magnitude at a distance of 7/8L from the rod's proximal end. The remaining three rods, one of each length, were unweighted. The addition of the .06$\mathrm{kg}$ weight to a rod changed its static moment relative to the pivot point defined by a "point" in the wrist.

\section{Apparatus}

The two experiments were conducted in a room that was partitioned by a styrofoam panel along its length, as depicted in Figure 1. Figure 1 shows a chair with an armrest at one end of the room. Between the chair and the opposite wall, there was a cardboard panel

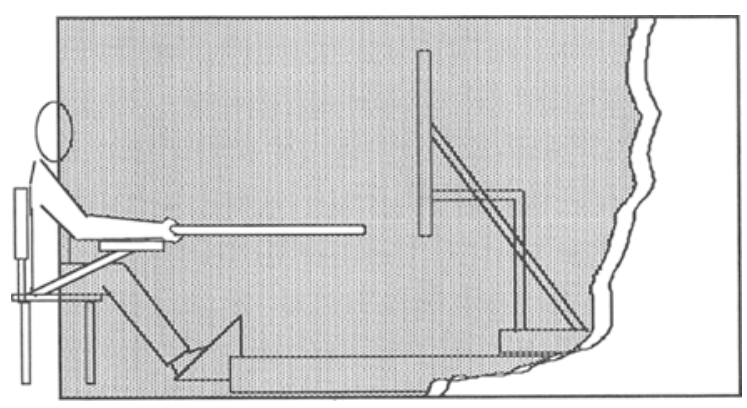

Figure 1. The apparatus used in the two experiments. Styrofoam screens (depicted in cutaway view) divided the area in two. Objects shaded in the diagram were behind the screens; as is evident, the subject's right arm, the rod, and the wrist support were on one side, and the rest of the subject, along with the tracks and the moving board, was on the other side. The subject was able to move the board by pressing foot pedals. 
perpendicular to the floor, mounted on a mobile platform. The platform was $61 \times 99 \mathrm{~cm}$. A subject seated in the chair could move the planar surface closer or farther away by controlling two foot pedals.

\section{Procedure}

In both experiments, the subject had to determine by effortful touch the effective reaching length of a rod held firmly in the right hand without movement. To enhance uniformity of grasp, all subjects in both experiments were instructed to keep the hand tight to the rod, and they were corrected if the rod was not fully enclosed by the hand. In both experiments, each subject's task was to indicate the perceived extent by positioning the visible vertical surface at a point where it could be reached with the hand-held rod. The partition (shown in cutaway view in Figure 1) precluded any visual inspection of the right arm and the rod.

The edge of the armrest of the chair was taken as the reference point for the measurement of the perceived extents. The haptically perceived reaching extent of a given rod was indicated by the selected position of the movable surface. This position was referred to a scale visible only to the experimenter. In the instructions, each subject was told (1) that the right arm and the rod would be visibly occluded throughout by the curtain; (2) to place the forearm on the armrest of the chair; and (3) to hold the rod still. The subject was encouraged to move the report board as much as he or she wanted. For each trial, the rod was placed in the subject's right hand so that the rod was close to parallel to the ground. A major criterion for the experimenter's manner of placing the rod in the hand was that it should not produce observable movement of the hand and rod. Trials in which there was discernible movement of the rod were repeated later in the experiment. ${ }^{1}$ There was a slight angle in the rod position when the rod was held (approximately $10^{\circ}$ to the ground, with the distal end raised relative to the proximal end), to prevent the back of the rod from contacting the armrest of the chair.

Each subject in the two experiments was given a demonstration of the task with a sample rod that was not part of the experimental set. After the demonstration, the subject was asked to keep his or her head and body, except for the right arm, behind the curtain for the duration of the experimental session. In Experiment 1, the subject was instructed to report how far he or she could reach with the part of the rod extending beyond the grasp. In other words, the subject had to judge the length of just the portion of the rod forward of the wrist. (When a rod was held at $\mathrm{H}=3 / 4 \mathrm{~L}$, most of the rod was behind the wrist.) In Experiment 2, the subject was instructed to report on how far he or she could reach with the rod if the rod were held at an end with its whole length extending forward of the wrist. In other words, the subject had to judge the length of the entire rod.

There were no practice trials in either experiment, nor was there any feedback during the course of either experiment. Within each session, each rod was held on several separate occasions. The ordering of rods across trials was random, with a different random ordering for each subject. The subjects in each experiment were different; no subjects, therefore, ever had experience with the task, and none were given the opportunity to practice it. The subjects were not informed about the number and length of the rods until they had completed the experiment.

\section{Design and Predictions}

The combination of three rod lengths, three rod weightings (none, at $1 / 8 \mathrm{~L}$, and at $7 / 8 \mathrm{~L}$ ), and two hand positions created 18 different conditions. In each experiment, rods were presented to the subject one at a time in a random order; all 18 conditions were run once before any condition was repeated. Each condition was run a total of three times to yield 54 trials per subject.

With respect to the first experiment, if the haptic subsystem can partition the rod into its component static moments, then for a rod of length $L$, the perception of the half length (that is, $H=1 / 2 L$ ) should be the same for the unweighted rod and for the rod with the weight attached at $1 / 8 \mathrm{~L}$; and the perception of the quarter length (i.e., $\mathrm{H}=3 / 4 \mathrm{~L}$ ) should be the same for the unweighted rod and for the rod with the weight attached at $1 / 8 \mathrm{~L}$. That is, the perception of the partial lengths forward of the position of the hand should be unaffected by the increase in static moment brought about by adding a weight below the position of the hand.

Similarly, if the haptic subsystem can separate the static moments above and below the fixed point, then for the rods with a weight added above the axis of rotation at $7 / 8 \mathrm{~L}$, the half lengths and quarter lengths should be perceived as longer than when the rods are unweighted. Let us refer to the three basic conditions of the experiment, with respect to the presence and position of the added weight, as follows: Condition $\mathrm{N}$ (for none), Condition 1/8, and Condition $7 / 8$. The following outcomes were expected, therefore, under the assumption that a subject could distinguish fractional static moments: (1) When the added weight is below the fixed point (the grasp), it should not affect the perception of the length above the grasp; that is, we expect the equality Condition $1 / 8=$ Condition $N$. (2) When the added weight is above the fixed point, it should affect the perception of the length above the grasp; that is, we expect the inequalities Condition $7 / 8>$ Condition $N$ and Condition $7 / 8>$ Condition $1 / 8$. (3) The perceived distances reachable with rods held at $H=1 / 2 \mathrm{~L}$ should be longer than the perceived distances reachable with rods held at $\mathrm{H}=3 / 4 \mathrm{~L}$.

Very different outcomes were expected for the second experiment: $A$ rod held at $H=1 / 2 \mathrm{~L}$ should be perceived as longer when weighted than when unweighted (because its total static moment is greater); so should a rod held at $\mathrm{H}=3 / 4 \mathrm{~L}$. That is, we expect the inequalities Condition $7 / 8>$ Condition $\mathrm{N}$ and Condition 1/8 $>$ Condition $N$. As for the comparison of Condition $1 / 8$ and Condition $7 / 8$, these should be perceived as equal in extent when the rods are held at $H=1 / 2 \mathrm{~L}$ (because the absolute value of the static moment is not altered by the added mass's being above or below the hand), and Condition 1/8 should be perceived as longer when the rods are held at $\mathrm{H}=3 / 4 \mathrm{~L}$ (because the distance between the pivot point and the added mass is $1 / 8 \mathrm{~L}$ when the mass is at $7 / 8 \mathrm{~L}$, and it is $5 / 8 \mathrm{~L}$ when the mass is at $1 / 8 \mathrm{~L}$ ).

\section{RESULTS}

For perception of partial lengths (Experiment 1), analysis of variance revealed significant effects of hand position $\left[F(1,7)=41.54, M S_{\mathrm{e}}=285.9, p<.001\right]$ and weight position $\left[F(2,14)=61.215, M S_{\mathrm{e}}=193.8, p<\right.$ $.001]$. The mean perceived partial lengths for the different hand positions were .611 and $.426 \mathrm{~m}$ for $\mathrm{H}=1 / 2 \mathrm{~L}$ and $\mathrm{H}=3 / 4 \mathrm{~L}$, respectively. Thus, the mechanically motivated expectation that rods held at halfway would be perceived as longer was confirmed. The means for the three weight conditions were $.463 \mathrm{~m}$ (no added weight), $.399 \mathrm{~m}$ (weight added at $1 / 8 \mathrm{~L}$, below the wrist), and $.694 \mathrm{~m}$ (weight added at 7/8L, above the wrist). As expected, there was a significant interaction between hand position and weight position $\left[F(2,14)=15.115, M S_{\mathrm{e}}=\right.$ $218.2, p<.001]$, reflecting the differing predictions for the rods held at $H=1 / 2 \mathrm{~L}$ and the rods held at $H=3 / 4 \mathrm{~L}$. The significant interaction between hand position and length of $\operatorname{rod}\left[F(2,14)=4.613, M S_{\varepsilon}=59.0, p<.05\right]$ was not expected; as is evident in Figure 2, this interaction results from the forward segment of the longest rod's being perceived as actually shorter than the forward seg- 
ment of the medium rod, when the rods were held at $\mathrm{H}=$ $3 / 4 \mathrm{~L}$. This effect is seen in 6 of the 8 individual subjects.

The interaction of weight condition and hand position is depicted in Figure 3. A Tukey honestly significant difference test supported the major predictions. For rods held at halfway, the means were $.509 \mathrm{~m}(\mathrm{~N}), .443 \mathrm{~m}$ $(1 / 8)$, and $.880 \mathrm{~m}(7 / 8)$; statistically, the rods with an added mass at $1 / 8 \mathrm{~L}$ and the rods with no added mass were not perceived as different, and each was perceived as significantly shorter than the rods with the mass added above the hand (for each, $p<.01$ ). For the rods held at $\mathrm{H}=$ $3 / 4 \mathrm{~L}$, the means were $.416 \mathrm{~m}(\mathrm{~N}), .354 \mathrm{~m}(1 / 8)$, and $.507 \mathrm{~m}(7 / 8)$; statistically, Condition $1 / 8$ was not significantly different from Condition $\mathrm{N}$, and was different $(p<.05)$ from Condition 7/8; Condition $\mathrm{N}$ was not different from Condition 7/8.

For Experiment 2, in which subjects reported the whole length of the held rods, analysis of variance revealed significant main effects of hand position $[F(1,5)=15.119$, $\left.M S_{\mathrm{e}}=115.0, p<.02\right]$, weight position $[F(2,10)=$ 18.164, $\left.M S_{\mathrm{c}}=538.0, p<.001\right]$, and rod length $\left[F(2,10)=21.783, M S_{e}=184.2, p<.001\right]$, as well as the expected interaction between hand position and weight condition $\left[F(2,10)=18.439, M S_{\mathrm{e}}=296.5, p<\right.$ $.001]$. No other interactions had significant effects. The means according to hand position were $.811 \mathrm{~m}$ for $\mathrm{H}=$ $1 / 2 \mathrm{~L}$ and $.892 \mathrm{~m}$ for $\mathrm{H}=3 / 4 \mathrm{~L}$, confirming the mechanically motivated prediction that rods held at $\mathrm{H}=3 / 4 \mathrm{~L}$ would be perceived as longer because of greater first moment. Note that the direction of this result is opposite that found for the same conditions when partial length was to be reported. For weight position, the means were $.676 \mathrm{~m}$

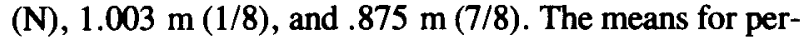

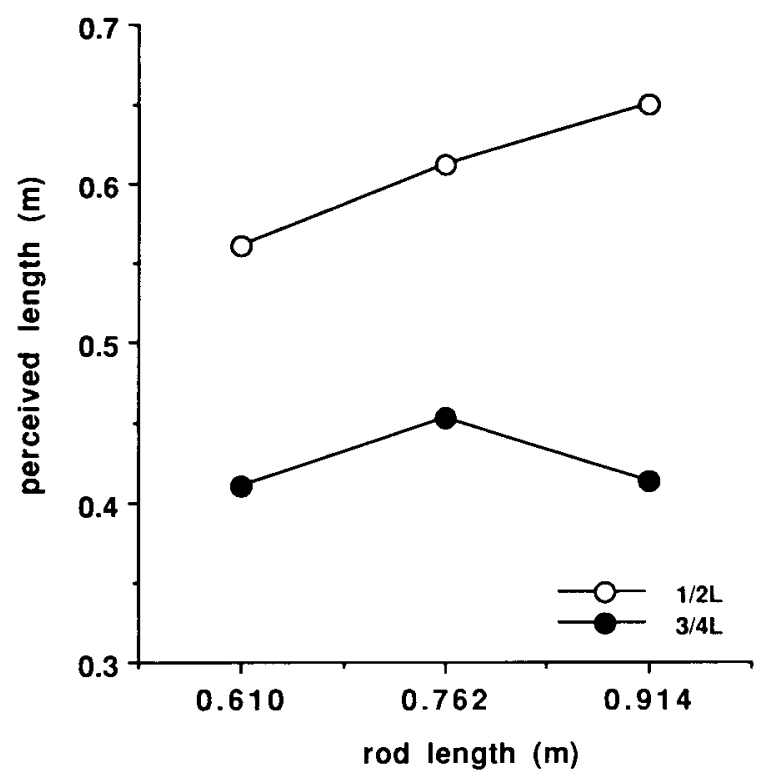

Figure 2. Perceived length (in meters) as a function of rod length and hand position in Experiment 1. Each data point represents 72 (8 subjects $\times 3$ weight positions $\times 3$ repetitions) judgments.

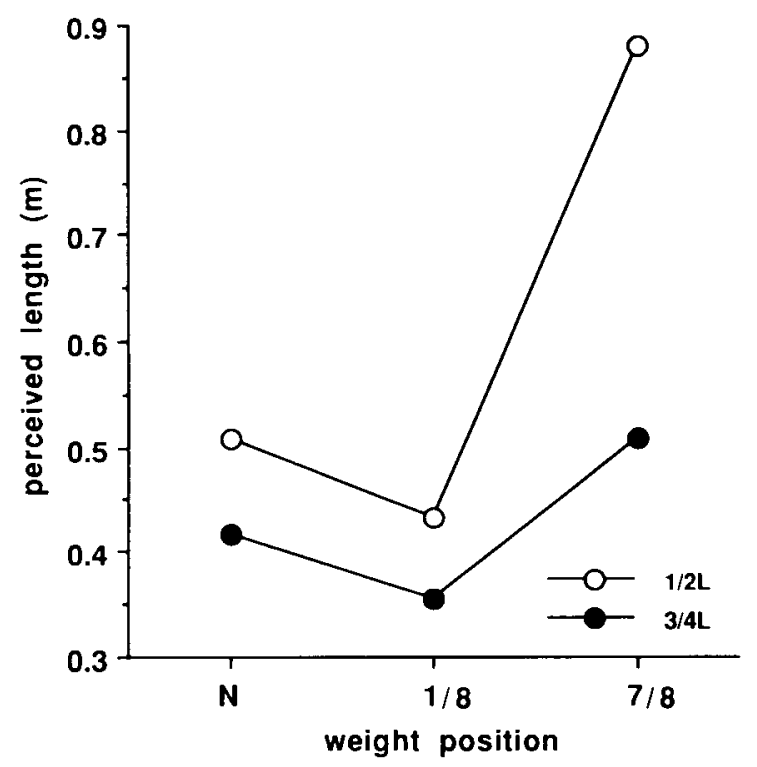

Figure 3. Perceived length (in meters) as a function of weight position and hand position in Experiment 1. Each data point represents 72 (8 subjects $\times 3$ rod lengths $\times 3$ repetitions) judgments.

ceived whole length were $.752, .841$, and $.962 \mathrm{~m}$, for the rods of lengths $.610, .762$, and $.914 \mathrm{~m}$, respectively.

The interaction between hand position and weight condition, shown in Figure 4, confirms the major predictions. For $\mathrm{H}=1 / 2 \mathrm{~L}$, the means were $.592 \mathrm{~m}(\mathrm{~N}), .868 \mathrm{~m}$ $(1 / 8)$, and $.974 \mathrm{~m} \mathrm{(7/8)}$. It made no significant difference whether the mass was added above or below the hand, but both of these conditions were significantly different from the condition with no added mass (each with $p<.01$, according to Tukey honestly significant difference tests). For the $\mathrm{H}=3 / 4 \mathrm{~L}$ conditions, the means were $.760 \mathrm{~m}(\mathrm{~N}), 1.138 \mathrm{~m}(1 / 8)$, and $.776 \mathrm{~m} \mathrm{(7/8)}$. The reported perceived lengths were significantly higher $(p<.01)$ when the weight was at $1 / 8 \mathrm{~L}$ than they were in the other conditions, while Conditions $7 / 8$ and $N$ were not different. The first moments of the rods in the latter conditions differed by $.003 \mathrm{~kg} \cdot \mathrm{m}$, so the lack of distinction is as expected (by way of comparison, the first moments of Conditions $7 / 8$ and $1 / 8$ differed by $.047 \mathrm{~kg} \cdot \mathrm{m}$ ).

It is apparent from the different orders of results across conditions in the two experiments that the subjects were able to perform the task differently under the different instructions, even though their explorations were minimized and thus could not have been specific to the different tasks (perception of partial length and perception of full length). The next set of analyses was designed to determine whether the mechanical variables constraining performance in the two experiments were indeed different, and whether these variables were appropriately specific to the partial or full extent of the rods. The first moment of mass distribution was computed for each of the conditions in two ways: as the static moment of the entire rod, and as the static moment of the portion of the 


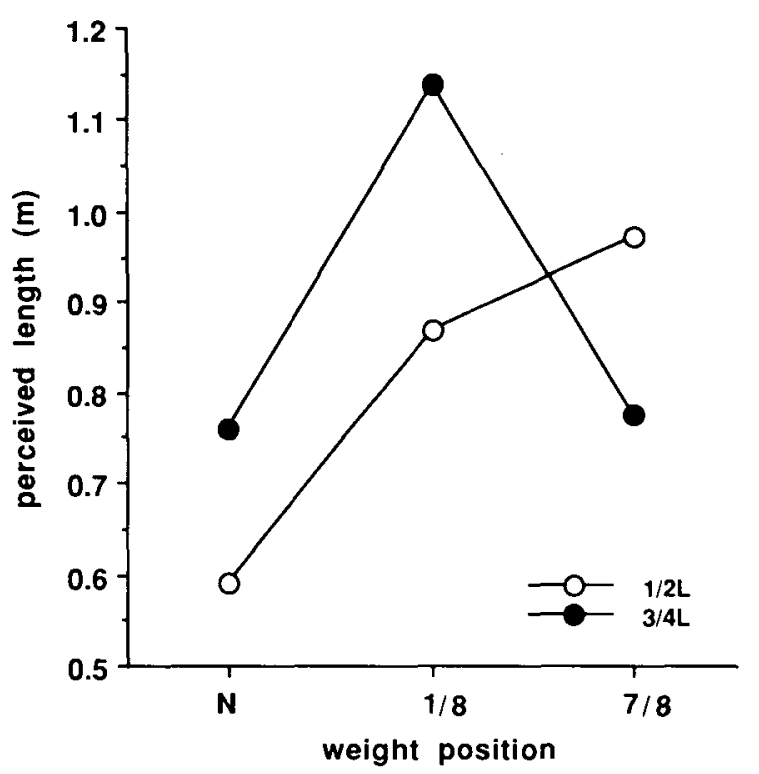

Figure 4. Perceived length (in meters) as a function of weight position and hand position in Experiment 2. Each data point represents 54 (6 subjects $\times 3$ rod lengths $\times 3$ repetitions) judgments.

rod forward of the wrist. The latter value was computed by deriving the first moment of a rod equal in length to the forward portion and held at its end.

Figure 5 shows the regression of perceived length against the appropriate moment variable: partial, for Experiment 1 (left panel), and full, for Experiment 2 (right panel). For comparison, the perceived partial length was also regressed against the first moment full, and the per- ceived full extent was also regressed against the first moment partial. In both cases, the amount of variance accounted for by the "inappropriate" static moment variables $\left(r^{2}=.001\right.$ and .04 , respectively) was lower than it was for the appropriate. In Figure 6, the perceived lengths in both experiments are shown together as a function of the associated moment variable. The data points for partial length are, naturally, those with the lower values for static moment. The slopes of the functions for partial and full lengths are slightly different, but nevertheless, despite the distinction in the portion of the rod perceived, the judgments in the two experiments follow the same curvilinear function of static moment of the appropriate portion.

From these results, it appears that the subjects, despite using minimal exploration, were constrained by different mechanical variables specific to the different instructions in the two tasks. Table 1 shows, for each subject individually, the results of multiple regression of the perceived partial lengths in Experiment 1 against two variables: first moment of the partial length, and first moment of the whole length. If subjects can indeed perceive the partial length distinct from the whole length, only partial variables should have an influence. The perceived lengths for all 8 subjects were significantly dependent on the combination of these mechanical variables. Moreover, all 8 subjects displayed a significant dependence on the first moment of the partial length, and only 2 showed any significant dependence on the first moment of the whole length. For these 2 subjects, the $F$ values for first moment of partial length are considerably higher. Table 2 gives the corresponding values for the perception of whole length. Here, the prediction would be reversed, and it
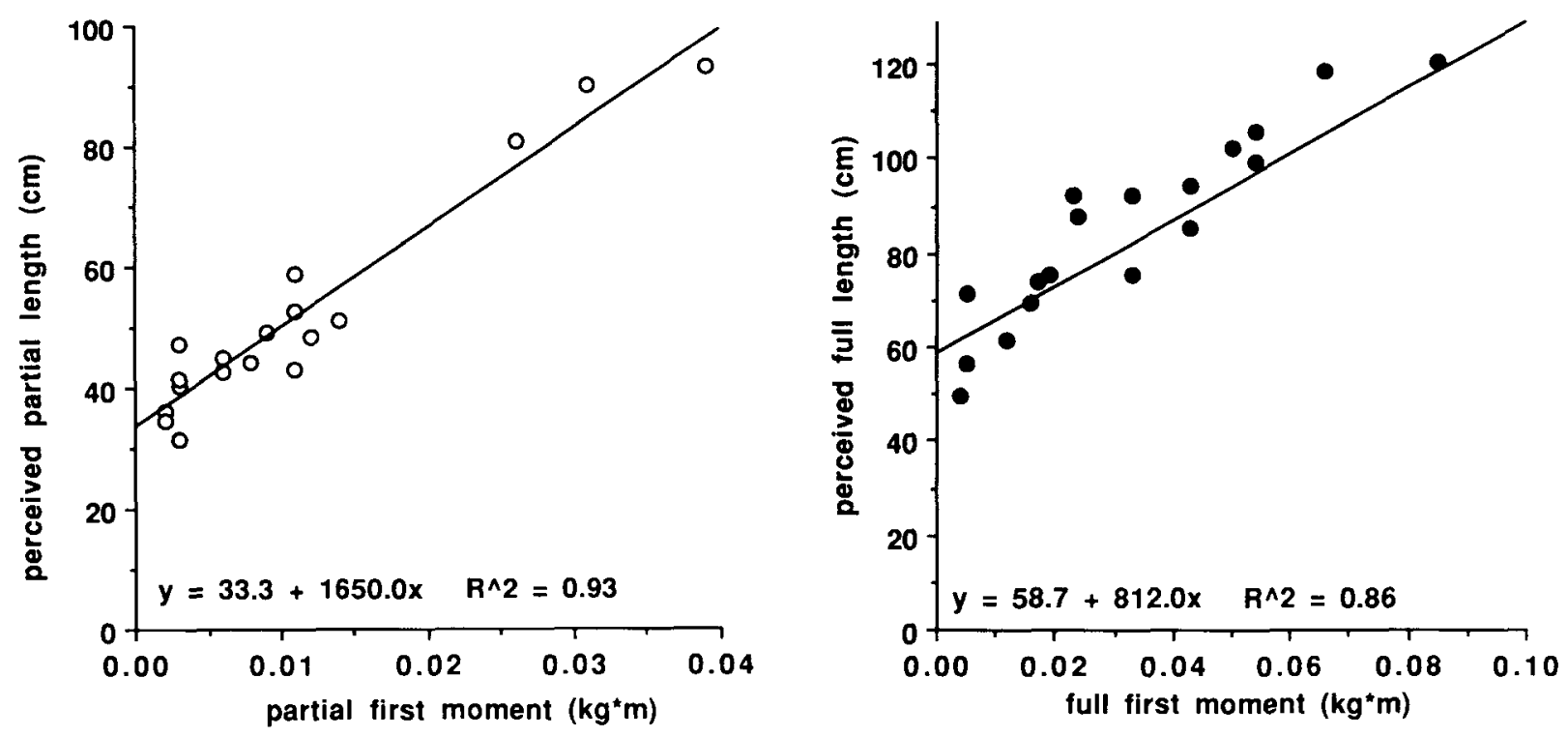

Figure 5. Perceived length (in meters) in the two experiments as functions of the first moment (in $\mathrm{kg} \cdot \mathrm{m}$ ) of the attended aspect of the rod. The left panel depicts the mean judgments of partial length as a function of the first moment of the partial length of the rod in Experiment 1; the right panel depicts the mean judgments of full length as a function of the first moment of the entire rod in Experiment 2. 


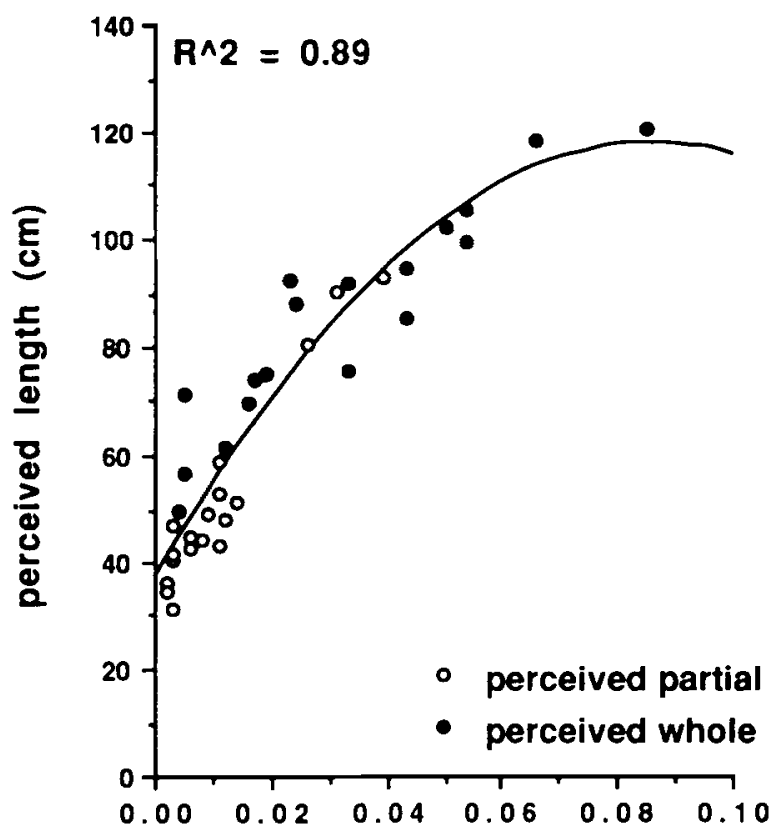

first moment $\left(\mathrm{kg}^{\star} \mathrm{m}\right)$

Figure 6. The perceived lengths in all conditions of both experiments together as a function of the associated first moment.

would be expected that the subjects' reports would be affected only by the static moment of the whole length. All 6 subjects showed a significant dependence on the combination, and likewise, the data for all subjects were significantly dependent on the first moment of whole length. Only 1 subject gave reports showing a dependence on partial first moment, but the $F$ value was lower than that for whole first moment.

It is possible to compare these partial $F$ s statistically by performing an analysis of variance on the standardized coefficients ( $\beta$-weights) associated with the computation of partial $F$ (see Burton \& Turvey, 1990, for another example of this procedure). The experimental instructions (judge partial or judge full length) were taken as a betweensubjects variable, and source of variance (first moment of partial length or first moment of full length) was entered as a within-subjects variable. If the relevance of these mechanical variables depends on the subjects' instructions, there should be an interaction: the first moment of the partial length should yield higher standardized coefficients under intention to perceive partial length, and the first moment of the full length should yield higher standardized coefficients under intention to perceive full length. This interaction was present $[F(1,12)=174.78]$, in the expected topography, and significant $(p<.0001)$. The main effects of instruction $[F(1,12)=4.71]$ and variable $[F(1,12)=5.43]$ were also significant $(p<.05)$. Specifically, the mean standardized coefficient was higher for partial moment than it was for full (.49 vs. .34), and it was higher for perception of full length than it was for perception of partial length (.46 vs. .37).

\section{DISCUSSION}

The two experiments differed only in terms of what had to be perceived. In Experiment 1, for a visually occluded rod grasped firmly and held steady at a position intermediate between its two ends, the subject's task was to perceive the distance reachable with the portion of the rod extending beyond the position of the grasp. In Experiment 2 , for a visually occluded rod similarly grasped firmly and held steady at a position intermediate between its two ends, the subject's task was to perceive the distance reachable with the entire rod if it were held at its proximal end. The results and analyses of the two experiments suggest that these two different measurement requirements were met in two different ways. In Experiment 1 , perceived extent was constrained by the mass distribution of the rod forward of the grasp about an axis through the proximal end of the rod segment; in Experiment 2 , perceived extent was constrained by the mass distribution of the entire rod about the given axis of rotation intermediate between the rod's ends. In sum, the two experiments provide evidence of selectivity in haptic perception resulting from the intention to attend to one property rather than another. We consider the significance of this evidence for the study of attentive phenomena in general and effortful or dynamic touch in particular.

\section{A Multiple Regression Technique for Investigating Selective Perception in Effortful Touch}

To begin with, the present experiments demonstrate the value of the technique of multiple regression as a general

Table 1

Partial $F$ Values Derived by Multiple Regressions of Perceived Length (in Meters) against First Moment (kg - $m$ ) of the Partial Length, and First Moment of the Full Length in Experiment 1

\begin{tabular}{ccc}
\hline Subject & Partial Moment & Full Moment \\
\hline 1 & $140.54 \ddagger$ & $7.46^{*}$ \\
2 & $45.70 \ddagger$ & .17 \\
3 & $138.68 \ddagger$ & 1.74 \\
4 & $53.41 \ddagger$ & .00 \\
5 & $28.75 \ddagger$ & 1.57 \\
6 & $20.64 \ddagger$ & 2.17 \\
7 & $117.68 \ddagger$ & $18.43 \ddagger$ \\
8 & $85.75 \ddagger$ & .20 \\
\hline 05 & $\dagger p<01$ &
\end{tabular}

Table 2

Partial $F$ Values Derived by Multiple Regressions of Perceived Length (in Meters) against First Moment (kg - m) of the Partial Length, and First Moment of the Full Length in Experiment 2

\begin{tabular}{rcc}
\hline Subject & Partial Moment & Full Moment \\
\hline 1 & .09 & $57.99 \dagger$ \\
2 & 2.71 & $67.03 \ddagger$ \\
3 & $5.20^{*}$ & $11.13 \dagger$ \\
4 & 2.69 & $56.70 \ddagger$ \\
5 & .49 & $63.27 \ddagger$ \\
6 & 3.35 & $28.76 \ddagger$ \\
\hline${ }^{*} p<.05$. & $\dagger p<.01$. & $\ddagger p<.001$.
\end{tabular}


method for determining and quantifying selectivity, and as a way to bring effortful or dynamic touch within the domain of attention research. The importance of this demonstration can be appreciated by reviewing some typical empirical strategies for studying selective attention in vision or audition. In one strategy, the observer tracks the value of a particular signal (e.g., he or she monitors a spatial location, repeats words presented to one ear, and so forth) in the context of various degradations and distractions. To the extent that the person can monitor exclusively one "channel" or stimulus type without intrusions from another or others, the person is said to demonstrate selectivity (see, e.g., Neisser \& Becklen, 1975). In another strategy, the issue is whether or not a subject has been influenced by stimuli presented on a channel that he or she has been asked to ignore. Selectivity is assessed by the rate of intrusions (see, e.g., MacKay, 1973). In yet another strategy, the availability of target information is minimized, and the question addressed is whether or not target perception is enhanced when attention is directed to a reduced portion of the region in which the targets are presented (see, e.g., Yantis \& Jonides, 1990).

Although the aforementioned strategies have provided insights into selective seeing and hearing, they are not well suited to the study of selective effortful touching. The properties discernible by effortful or dynamic touch are not easily manipulated in the continuous fashion required by the tracking strategy. For example, the mass distribution of a wielded object cannot be varied in the course of wielding as conveniently as the pitch of a stream of sound can be varied in the course of listening. The strategy of counting intrusions is also ill suited to effortful or dynamic touch, given that mechanical properties-in contrast to the letters, numbers, and words commonly used in visual and auditory attention research-do not submit to discrete characterizations. ${ }^{2}$ Finally, insufficient understanding of what constitutes a "brief exposure" for effortful touch, and skepticism that such an analogue has any realization in this perceptual domain (Neisser, 1976), render the tachistoscopic, reduced stimulation strategy inadvisable (although it may be applicable in other touch situations; see Loomis \& Lederman, 1986).

In contrast to the more established strategies, the multiple regression strategy employed in the present study and in other related studies (Burton \& Turvey, 1990; Chan \& Turvey, 1991; Solomon et al., 1989b) suggests a way to open up the phenomena of effortful touch, and those of haptic perception more generally, to attentional issues. (Reviews of theories and experimental results in the field of attention [e.g., Allport, 1990; Broadbent, 1970; Johnston \& Dark, 1986; Moray, 1969] report no previous investigations of selective effortful touch. Studies of selective tactile perception do exist [e.g., Critchley, 1949; Korin \& Fink, 1959], but these pertain to clinical observations of abnormal conditions. The haptic percep- tion studies of Lederman and Klatzky [1987, 1990], in which distinct broad classes of hand and finger movements are associated with the intention to judge distinct object properties, might also be mentioned-although the authors do not specifically discuss their results in terms of selective attention. See also the studies from the same laboratory that are mentioned in note 2.) Investigating attention within the haptic subsystem of effortful touch may have special advantages, given the intimate connection between this subsystem's capabilities and the well-established facts of statics and dynamics. Ultimately, effortful or dynamic touch is constrained by forces, torques, impulse forces, and impulse torques, and by the mechanical parameters, such as the moments of the mass distribution, that are constant over the force variations. The study of selectivity within the haptic perceptual system can therefore benefit from the sophisticated theory and mathematical methods developed to address the coupling of mechanical forces to motions.

\section{Possible Covert Attention}

In previous experiments, researchers have demonstrated the ability of subjects to perceive whole and partial extents haptically under conditions of free wielding about a "point" in the wrist (Solomon \& Turvey, 1988; Solomon et al., 1989b). The comparison of the two experiments of the present article strongly suggests that people can partition haptically the mass distribution of a handheld rod in the absence of the opportunity to systematically vary the patterning of torques and, therefore, the patterning of tissue deformations. Even with the overt aspects of selective effortful touching reduced to a minimum (holding without wielding), a person can still perceive either the rod in its entirety or just the part forward of the hand. In the future, some haptic perception analogue to the monitoring of eye movements might help illuminate the extent to which the selectivity in the current situation is truly covert. For now, the statistical evidence is quite suggestive of relatively covert attention.

The ability of people to attend selectively and covertly to one of two spatially and temporally overlapping figures or events has been demonstrated in a number of experiments on visual perception (Neisser \& Becklen, 1975; Nicoletti \& Umiltá, 1989). Such an object/event configuration would pattern the optical distribution so that it is simultaneously informative about both components. It has also been demonstrated that attending to a specific location in a frontal, perpendicular display partitions the display into distinct left and right regions about the attentional focus rather than about the midline of the body (Nicoletti \& Umiltá, 1989). The results of the two experiments reported here, and those of Solomon and Turvey (1988) and Solomon et al. (1989b), suggest that closely similar selective attention and partitioning abilities are to be found in the domain of effortful touch (and, one might suspect, even more generally). It is worthwhile, 
therefore, to consider the implications of the haptic capability identified in the previous sections.

\section{Defining the Problem of Covert Attention in Effortful Touch}

Because effortful or dynamic touch is affected only by mechanical stimulation, the task of perceiving lengths must be grounded in one or more mechanical variables that include the dimension of mass in their definition. Length is not such a variable. Consequently, a perceptual measure of the length of rod segment forward of the hand must be related in some fashion to the mass distribution of that segment, simply because there is no other way to gain an awareness of extent by effortful touching. The results of the experiments concur with this assertion.

The torque deforming the tissues is $N=M R \times g$, and it would be of the same magnitude and direction for a given rod held with the intent to perceive the whole extent of the rod or the extent of the rod segment forward of the hand. The experiments, therefore, pose a challenge with respect to understanding how different perceivings can be achieved under the partial and whole instructions, given apparently identical conditions of torque and tissue deformation.

In physical terms, the perceptual ability to segment the mass distribution of an object inverts the normal principle governing the determination of $M R$ for an object. An object is said to consist of many particles (of the same mass if the object is materially homogeneous) at different distances from an arbitrarily chosen origin. Each particle is acted upon by a vertical weight force $d M g$. A major theorem of statics tells us that we can replace the many vertical, parallel forces by one force (e.g., den Hartog, 1948). The magnitude of this resultant about the origin is the sum of all the component forces taken algebraically, $M g=g \int d M$; this single force, when acting alone, produces the same effect as the simultaneous action of its components. Furthermore, by the same theorem, the moment of the resultant about the origin is equal to the sum, taken algebraically, of all the moments of the component parallel forces, $M g R=g \int d M r$; this single moment, when acting alone, produces the same effect as the simultaneous action of its components. In the present experiments, the origin of the coordinate system about which $g \int d M r$ is defined is in the vicinity of the hand's position on the rod. (We presume that it is the pivot point in the wrist.) The results of the experiments suggest an inversion of the principle of equivalence of moments (Solomon et al., 1989b): Given the resultant of the many component momentsthe weight force $M g$ acting through the rod's center of gravity at a distance $\boldsymbol{R}$ from the origin-it is as if the subjects decomposed that resultant into specific component moments, those corresponding to the mass distributions of the rod fore and aft of the origin, or $g M R=g \int d M r_{\text {fore }}$ $+g \int d M r_{\text {aft }}$.

Patently, the perceptual measure of rod extent cannot involve the actual dissecting of the rod and calculation of $\int d M r_{\text {fore }}$. Furthermore, the proposal of a general algorithm for decomposing any given $M g R$ must be viewed with skepticism, given that there are no physical principles upon which such an algorithm could be based; mechanically, an infinite number of components would sum to a particular resultant, just as $4+5$ has only one result but many addends sum to 9 . So how is $\int d M r_{\text {fore }}$ made available to the perceptual system, and how is the perceptual measure of forward extent thereby achieved? The answer must be that there is a patterning of tissue deformation specific to $\int d M r_{\text {fore }}$. By way of sketching the direction of possible future research, let us consider two possible candidates.

1. Are there anatomical touch "cues"? Depending on where the rod was held, on whether or not there was an additional mass, and on where the additional mass was placed, the rod in the hand would produce a pressure pattern that could be completely downward (a uniform rod held at its midpoint), or downward in the forward part of the hand and upward in the backward part of the hand (a rod held at its midpoint with a mass attached ahead near its distal tip), or vice versa (a rod held at its $3 / 4$ point with a mass attached behind near its proximal tip). Possibly, the attentional capabilities evident in the two experiments might be interpretable as focusing on the overall pressure distribution in order to perceive whole extent, and focusing on a particular part of the distribution in order to perceive partial extent. That is, a subject might distribute attention over the pressure pattern in different ways, depending on whether he or she was trying to perceive the whole rod or the forward segment (analogous to the subjects of Tsal and Kolbert [1985] who perceived a duck or a rabbit, depending on where in the ambiguous figure attention was focused). There may be some truth to this way of thinking about the problem, but it is unlikely that any simple feature of a given region of pressure on the skin can constrain the perception of partial extent. We cannot expect to find that the pressure on, say, the second metacarpal is a good "cue" for segment length. For the perception of extents forward of the hand, the rod conditions of hand at $1 / 2 \mathrm{~L}$ and no added mass, hand at $3 / 4 \mathrm{~L}$ and no added mass, hand at $1 / 2 \mathrm{~L}$ and added mass at $1 / 8 \mathrm{~L}$, and hand at $3 / 4 \mathrm{~L}$ and added mass at $7 / 8 \mathrm{~L}$, for example, would produce very different patternings of pressure at and around the second metacarpal and, for that matter, on all parts of the hand. Moreover, previous research suggests strongly that the facility evident in the present experiments generalizes to other grips involving pressure distributions over other parts of the hand (e.g., the palm, when the rod is gripped with the palm facing upward), and considerably different stress, shear, and torsional forces on the forearm musculature (Carello, Fitzpatrick, Domaniewicz, Chan, \& Turvey, in press; Hoisington, 1920; Solomon et al., 1989a). In sum, a solution couched in terms of any particular local pressure feature is not likely to succeed. The basis for the ability must be sought in more global and general properties of the rod-induced deformation pattern.

A similar but perhaps more plausible possibility is that, in the near absence of exploratory movements of the rod, the style of the very act of grasping the rod might be dis- 
tinct under the intention to perceive distinct properties. As detailed in the introduction, the hand is hardly passive in the current situation, not even if it were possible for the hand to be completely immobile, because the hand and wrist must produce the force that prevents the rod from moving. Could the subject be adapting his or her grip style to the intended perceptual object, grasping the rod in one way to perceive full length and in another way to produce partial length? Such a possibility could be tested either by orthopedically immobilizing the wrist to prevent even these variations in holding, or by conducting subjects who alternately judge both partial length and full length and placing the rods in the subject's hand before the subject is aware of which property is to be judged. ${ }^{3}$ The latter strategy could be profitably linked to analysis of recordings of the grasp, because presumably this procedure would be as prone to the "haptic noise" of small movements of the rod as was the procedure in the present experiments, and we would want to satisfy ourselves that these movements did not constitute rearrangement of the grasp. Although these measures could well repay the effort, we have not pursued them here for mechanical reasons. To reiterate the physical analysis in the introduction, the torque that must be applied by the hand and wrist to hold still a hand-held object is determined by the mechanical dimensions of the object and the location of the grasp. We assume that the deformation patterns induced by these torques are informative-particularly their invariant aspect tied to the first moment. If this assumption is right, the informative component of the grip is determined by the properties of the rod; no informative variables of the grip remain that could be varied under different intentional conditions.

2. Is there information in differential compression/ shear? Holding a rod in the hand roughly parallel to the ground, with the forearm resting on a support, bends the wrist in the manner depicted in Figure 7 . We may, for purely illustrative purposes, consider the hand as a beam fixed to a surface (the distal surfaces of the radius and ulna bones), with a load at its free end (the rod in the grasp). The hand and wrist thus suggest a cantilever bent only in the vertical plane, where the bend is exaggerated. The external loading provides a bending moment that induces internal forces. If we consider only tensile and compressive stresses and the resultant longitudinal strains, tissues above the midline $\mathrm{OO}^{\prime}$ are stretched and tissues below the midline are shortened. There is a gradient of elongation from zero to a maximum, and a gradient of shortening from zero to a maximum. As the center of mass of the rod varies with respect to $O$ (see Figure 7), the tensile and compressive stresses will change systematically, as will the gradients of lengthening and shortening. Given even this limited depiction of the structuring of strains, one can see how questions can be raised about variables of tissue deformation that might be specific to how the mass is distributed forward and behind the hand. Speculatively, the relation between the tensile and compression gradients could be such a variable.
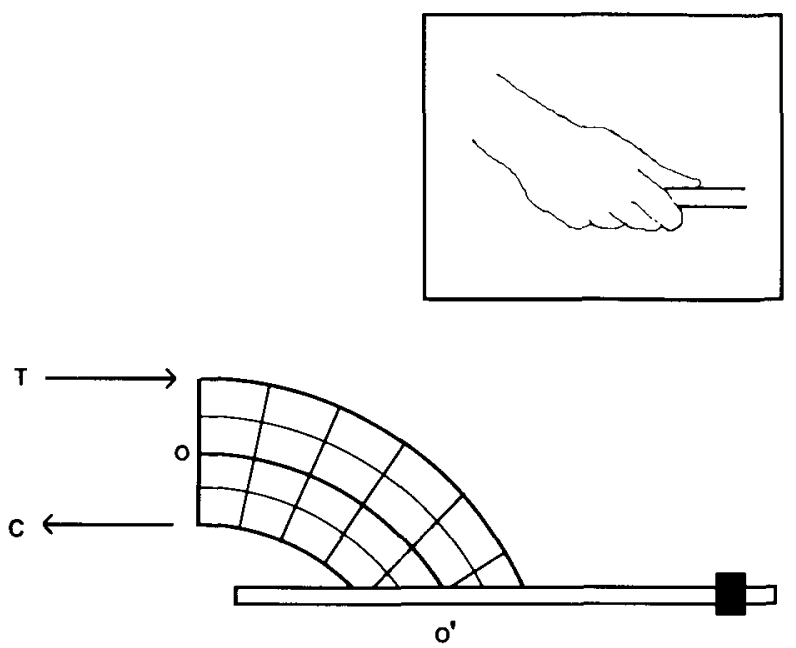

Figure 7. The exaggerated cantilever model. Under a load, material above the midline $\left(\mathrm{OO}^{\prime}\right)$ will undergo tensions relative to the midline, and material below the midline will undergo compressions. Inside panel shows the attitude of the hand. See text for details.

\section{Concluding Remarks}

In sum, the evidence for attentionally splitting the mass distribution of a hand-held rod with minimal effortful touch raises two questions for future investigations: Are there deformation patterns differentially specific to $\int d M r_{\text {fore }}$, $\int d M r_{\mathrm{aft}}$, and $M R$ ? And if there are, how can perception be constrained selectively by them?

With respect to the first question, an enterprise of analyzing haptic information as a structured array of strains when effortful touching is minimal (and as a structured array of rates of strain when effortful touching is time-varying) would have much in common with efforts to identify informative structures in optic flow through differential invariants (see, e.g., Koenderink \& van Doorn, 1978; Waxman \& Ullman, 1985). As Solomon (1988) and Solomon et al. (1989a) have noted, it is possible that optic and haptic information can be treated in similar ways, as sharing similar specifying variables (see also Kugler \& Turvey, 1987).

With respect to the second question, an account of selective perception in effortful touching is being pursued, in which it is assumed that (1) the haptic perceptual system can function in a number of special-purpose ways, and (2) prior intents are functionally equivalent to physical constraints that harness dynamics to form task-specific mechanisms (see, e.g., Solomon, 1988; Solomon \& Turvey, 1988; Turvey, 1986, 1988). The key idea is that intention causes an organization of the haptic perceptual system specially suited to the extraction of information about the property of interest; in turn, the extracted information causes a specific haptic perceptual experience (of the property of interest). There are currently a number of theoretical and experimental analyses of perception and action systems as softly assembled, special-purpose, smart devices (e.g., Bingham, 1988; Bingham, Schmidt, \& 
Rosenblum, 1989; Schöner \& Kelso, 1988; Solomon, 1988) and of intention harnessing dynamics (e.g., Kugler \& Turvey, 1987; Schöner \& Kelso, 1988). It remains to be seen whether these new directions can lead to a deeper understanding of the phenomenon of selective perception in effortful touch and of the phenomenon of attention more generally.

\section{REFERENCES}

Allport, A. (1990). Visual attention. In S. Posner (Ed.), Foundations of cognitive science (pp. 631-682). Cambridge, MA: Bradford Books, MIT Press.

Bingham, G. (1988). Task specific devices and the perceptual bottleneck. Human Movement Science, 7, 225-264.

Bingham, G., Schmidt, R. C., \& Rosenblum, L. R. (1989). Hefting for a maximum distance throw: A smart perceptual mechanism. Journal of Experimental Psychology: Human Perception \& Performance, 15, 507-528.

Brondbent, D. E. (1970). Stimulus set and response set: Two kinds of selective attention. In D. I. Mostofsy (Ed.), Attention: Contem porary theories and analysis (pp. 51-60). New York: AppletonCentury-Crofts

Burton, G., \& Turvey, M. T. (1990). Perceiving the lengths of rods that are held but not wielded. Ecological Psychology, 2, 295-324.

Carello, C., Fitzpatrick, P., Domaniewicz, I., Chan, T.-C., \& TurveY, M. T. (in press). Effortful touch with minimal movement. Journal of Experimental Psychology: Human Perception \& Performance.

Chan, T.-C., \& Turvey, M. T. (1991). Perceiving the vertical distances of surfaces by means of a hand-held probe. Joumal of Experimental Psychology: Human Perception \& Performance, 17, 347-358.

Critchley, M. (1949). The phenomenon of tactile inattention with specific reference to parietal lesions. Brain, 72, 538-561.

DeN Hartog, J. P. (1948). Mechanics. New York: Dover.

Driver, J., \& BAyliss, G. C. (1989). Movement and visual attention: The spotlight metaphor breaks down. Journal of Experimental Psychology: Human Perception \& Performance, 15, 448-456.

GiBson, J. J. (1966). The senses considered as perceptual systems. Boston: Houghton-Mifflin.

GiBson, J. J. (1979). The ecological approach to visual perception. Hillsdale, NJ: Erlbaum.

Goryo, K., Robinson, J. O., \& WiLson, J. A. (1984). Selective looking and the Müller-Lyer illusion: The effect of changes in the focus of attention on the Müller-Lyer illusion. Perception, 13, 647-654.

Hoisington, L. B. (1920). On the non-visual perception of the length of lifted rods. American Journal of Psychology, 31, 114-146.

Johnston, W. A., \& DARK, V. J. (1986). Selective attention. Annual Reviews of Psychology, 37, 43-76.

KlatzKy, R. L., Lederman, S. J., Re Red, C. (1989). Haptic integration of object properties: Texture, hardness, and planar contour. Journal of Experimental Psychology: Human Perception \& Performance, 15, 45-57.

Koenderink, J. J., \& VAN Doorn, A. (1978). How an ambulant observer can construct a model of the environment from the geometrical structure of the visual inflow. In G. Hauske \& E. Butenandt (Eds.), Kybernetik 1978 (pp. 224-247). Munich: Oldenberg.

KorIN, H., \& FINK, M. (1959). The role of set in the perception of simultaneous tactile stimuli. American Joumal of Psychology, 72, 384-392.

Kugler, P. N., Turvey, M. T. (1987). Information, natural law, and the self-assembly of rhythmic movement. Hillsdale, NJ: Erlbaum.

Lederman, S. J., Browse, R. A., \& Klatzky, R. L. (1988). Haptic processing of spatially distributed information. Perception \& Psychophysics, 44, 222-232.

Lederman, S. J., \& KLATZKY, R. L. (1987). Hand movements: A window into haptic object recognition. Cognitive Psychology, 19, 342-368.

LederMAN, S. J., KLATZKY, R. L. (1990). Haptic classification of common objects: Knowledge-driven exploration. Cognitive Psychology, 22, 421-459.
Loomis, J. M., \& Lederman. S. J. (1986). Tactual perception. In K. R. Boff, L. Kaufman, \& J. P. Thomas (Eds.), Handbook of perception and human performance: Vol. 2. Cognitive processes and performance (pp. 31/1-31/41). New York: Wiley.

MACKAY, D. G. (1973). Aspects of the theory of comprehension, memory, and attention. Quarterly Joumal of Experimental Psychology, 25, 22-40.

Moray, N. (1969). Listening and attention. Baltimore, MD: Penguin. NeISSER, U. (1976). Cognition and reality. San Francisco: W. H. Freeman

Neisser, U., \& Becklen, R. (1975). Selective looking: Attending to visually specified events. Cognitive Psychology, 7, 480-494

Nicoletti, R., \& Umiltá, C. (1989). Splitting visual space with attention. Journal of Experimental Psychology: Human Perception \& Performance, 15, 164-169.

SChÖNER, G.. Kelso, J. A. S. (1988). Dynamic patterns of biological coordination: Theoretical strategy and new results. In J. A. S. Kelso, A. J. Mandell, \& M. F. Shlesinger (Eds.), Dynamic pattems in complex systems (pp. 77-102). Singapore: World Scientific.

Solomon, H. Y. (1988). Movement-produced invariants in haptic exploration: An example of a self-organizing, information-driven, intentional system. Human Movement Science, 7, 201-224.

Solomon, H. Y., \& TuRveY, M. T. (1988). Haptically perceiving the distance reachable with hand-held objects. Joumal of Experimental Psychology: Human Perception \& Performance, 14, 404-427.

Solomon, H. Y., Turvey, M. T. Burton, G. (1989a). Gravitational and muscular variables in perceiving extent by wielding. Ecological Psychology, 1, 265-300.

Solomon, H. Y., Turvey, M. T., Burton, G. (1989b). Perceiving extent of rods by wielding: Haptic diagonalization and decomposition of the inertia tensor. Joumal of Experimental Psychology: Human Perception \& Performance, 15, 58-68.

TSAL, Y. (1984). A Müller-Lyer illusion induced by selective attention. Quarterly Joumal of Experimental Psychology, 36A, 319-333.

TSAL, Y., KolBERT, L. (1985). Disambiguating ambiguous figures by selective attention. Quarterly Journal of Experimental Psychology, 37A, 25-37.

TURVEY, M. T. (1986). Intentionality: A problem of multiple reference frames, specificational information, and extraordinary boundary conditions on natural law. Behavioral \& Brain Sciences, 9, 153-155.

Turvey, M. T. (1988). Simplicity from complexity: Archetypal action regimes and smart perceptual instruments as execution-driven phenomena. In J. A. S. Kelso, A. J. Mandell, \& M. F. Shlesinger (Eds.), Dynamic patterns in complex systems (pp. 327-347). Singapore: World Scientific.

Turvey, M. T., Solomon, H. Y., \& Burton, G. (1989). An ecological account of knowing by wielding. Joumal of the Experimental Analysis of Behavior, 52, 397-407.

WAXMAN, A., \&llman, U. (1985). Surface structure and threedimensional motion from image flow kinematics. Intemational Joumal of Robotics Research, 4, 72-94.

YANTIS, S., \& JoNidES, J. (1990). Abrupt visual onsets and selective attention: Voluntary versus automatic allocation. Joumal of Experimental Psychology: Human Perception \& Performance, 16, 121-134.

\section{NOTES}

1. It should be emphasized that the claim that exploration is minimized in the current experiments is not based on trust in the subjects' ability to comply with these instructions. The minimization of exploration can be evaluated empirically because of the mechanical fact that moment of inertia, being resistance to rotation, is undefined when there is no rotation. To the extent that movement of the rod has been reduced, the participation of moment of inertia must also be reduced. In Burton and Turvey (1990), the correlation between first moment and second moment was broken, and the power of moment of inertia to predict perceived length judgments was indeed significantly reduced under instructions identical to those in the present experiment. This observation is important, because no claim is made for the present experiment that subjects held the rods without any movement whatsoever, a condition that would be impossible without orthopedic restraint. The results of Burton and Turvey (1990) show that the small unavoidable movements 
are not extensively used by subjects to pick up information inaccessible under ideally motionless exploration. In the present experiment, it was not possible to compare the involvement of first moment and moment of inertia because they were highly correlated $\left(r^{2}=.95\right.$ for partial lengths and .89 for full lengths).

2. Some of the techniques successfully employed by Lederman and her colleagues in the case of haptic touch could be considered versions of the intrusion strategy. For example, subjects in the experiments of Klatzky, Lederman, and Reed (1989) were instructed to sort unseen objects that differed in both texture or hardness, by using only one of the two dimensions. When the redundancy was broken, sorting speed declined even when the diagnosticity of the dimension to be attended was unchanged. Lederman, Browse, and Klatzky (1988) assessed the interference between object texture and object orientation by requiring subjects to respond to haptic stimuli with particular combinations of values.

3. We are grateful to an anonymous reviewer for pointing out the grip as a potential site for exploratory distinctions and for the basic suggestions about how this possibility could be investigated.

(Manuscript received August 17, 1990; revision accepted for publication March 4, 1991.) 